\title{
Fat mass and obesity-associated (FTO) rs9939609 polymorphism modifies the relationship between body mass index and affective symptoms through the life course: a prospective birth cohort study
}

\author{
Shinsuke Koike ${ }^{1,2,3}$, Marcus Richards' ${ }^{1}$, Andrew Wong (D) $^{1}$ and Rebecca Hardy ${ }^{1}$
}

\begin{abstract}
Although bi-directional relationships between high body mass index (BMI) and affective symptoms have been found, no study has investigated the relationships across the life course. There has also been little exploration of whether the fat mass and obesity-associated (FTO) rs9939609 single-nucleotide polymorphism (SNP) is associated with affective symptoms and/or modifies the relationship between BMI and affective symptoms. In the MRC National Survey of Health and Development (NSHD), 4556 participants had at least one measure of BMI and affective symptoms between ages 11 and 60-64 years. A structural equation modelling framework was used with the BMI trajectory fitted as latent variables representing BMI at 11, and adolescent (11-20 years), early adulthood (20-36 years) and midlife (36-53 years) change in BMI. Higher levels of adolescent emotional problems were associated with greater increases in adult BMI and greater increases in early adulthood BMI were associated with higher subsequent levels of affective symptoms in women. The rs9939609 risk variant (A allele) from 2469 participants with DNA genotyping at age 53 years showed mostly protective effect modification of these relationship. Increases in adolescent and early adulthood BMI were generally not associated with, or were associated with lower levels, of affective symptoms in the FTO risk homozygote (AA) group, but positive associations were seen in the $\Pi$ group. These results suggest bi-directional relationships between higher BMI and affective symptoms across the life course in women, and that the relationship could be ameliorated by rs9939609 risk variant.
\end{abstract}

\section{Introduction}

Obesity and affective symptoms are common conditions; the life-time prevalence of each is estimated to be greater than $20 \%$ in the general population in highincome and middle-income countries ${ }^{1,2}$. Both increase the risk of physical and psychiatric diseases such as

\footnotetext{
Correspondence: Shinsuke Koike (skoike-tky@umin.ac.jp)

${ }^{1}$ MRC Unit for Lifelong Health and Ageing at University College London, 33 Bedford Place, London WC1B 5JU, UK

${ }^{2}$ University of Tokyo Institute for Diversity \& Adaptation of Human Mind

(UTIDAHM), 3-8-1 Komaba, Meguro-ku, Tokyo 153-8902, Japan

Full list of author information is available at the end of the article
}

cardiovascular diseases, cerebrovascular diseases, diabetes mellitus, and major depressive disorders ${ }^{1-4}$. In addition, among those who develop disease, obesity and depression are associated with poor prognosis and increased mortality $^{2-4}$. Obesity and depression, however, exhibit different patterns across the life course. The prevalence of obesity increases with age until the seventh decade ${ }^{5}$, while the pattern is less clear for affective symptoms. A recent study showed increases during adolescence and decline thereafter ${ }^{6}$, with a gender difference in the prevalence of depression evident from the beginning of adolescence to late adulthood ${ }^{7}$. 
A meta-analysis of longitudinal studies of adults showed that overweight and obesity at baseline were associated with an increased risk of depression at follow-up, and that depression at baseline was associated with subsequent higher risk of obesity, but not overweight ${ }^{8}$. Similar bidirectional associations between higher body mass index (BMI) and depressive symptoms have also been found ${ }^{9-12}$, especially in women ${ }^{9,10,12}$. Multiple possible explanations have been proposed for the relationship between higher body weight and depressive symptoms. Shared biological mechanisms such as immunological deficiencies, oxidative stress, neurotransmitter imbalance, and hypothalamic-pituitary-adrenal (HPA) imbalance are likely to play a role ${ }^{13-15}$. This is also likely to be the case for shared developmental and early life environmental risk factors such as low birth weight, perinatal complications, maternal depression, parental socioeconomic status, low childhood cognition, childhood trauma, and chronic stress; ${ }^{13,14,16}$ and shared later life risk symptoms such as insomnia and sleep apnoea ${ }^{15,17}$. Changes in health-related behaviour, such as decreased physical activity, disturbance of eating style, and also decreased self-esteem, are likewise common to both affective symptoms and obesity ${ }^{13,14}$. The relationship between BMI and affective symptoms may change through the life course and be dependent on age at onset of affective symptoms and $\operatorname{sex}^{12}$. We previously found that women with adolescent affective symptoms but no adults symptoms had lower BMI in adolescence but greater increases in BMI in adults ${ }^{12}$. Whereas men with adolescent symptoms which continued into adulthood had lower BMI in adolescence and across adulthood. However, no study has investigated the bi-directional relationship between BMI and affective symptoms across the life course.

Genetic studies have repeatedly reported the association between a common variant in the fat mass and obesityassociated (FTO) SNP rs9939609 and increased BMI ${ }^{18-21}$. In addition, a meta-analysis of randomized controlled trials suggested that those carrying the rs9939609 risk allele responded equally well to weight loss interventions as those without the risk allele ${ }^{21}$. Although the mechanism and function of $F T O$ is not fully understood, possible functional alterations by the gene have been reported in the HPA axis and in the reward system in the brain ${ }^{22-24}$. Disturbances of these systems have also been reported in major depressive disorders, which could lead to several core symptoms such as aberrant emotional and reward responses ${ }^{4,13}$. A prospective cohort study showed an association between another FTO SNP rs1421085, which has a strong linkage disequilibrium with the SNP rs9939609, and self-reported depressive symptoms in men, independent of $\mathrm{BMI}^{25}$. A clinical case-control study showed an association between the SNP rs9939609 and BMI only for people with major depressive disorder while no association was observed among healthy controls ${ }^{26}$. This is supported by a recent meta-analysis suggesting that affective status modifies the association between the FTO genotype and obesity ${ }^{27}$. However, the association may not be seen in Asian populations ${ }^{28}$, and another casecontrol study including five different ethnicities provided evidence of a protective effect of the rs9939609 obesityrelated allele on major depression independent of $\mathrm{BMI}^{29}$. There is no longitudinal study which has investigated whether associations between FTO and affective symptoms vary over the life course, or whether they may modify the relationship between BMI and affective symptoms.

The Medical Research Council National Survey of Health and Development (NSHD), also known as the 1946 British birth cohort, is one of the longest-running prospective cohort studies, which has repeatedly measured body height and weight throughout life, and obtained affective symptoms over a 40 -year period. We previously reported that the relationship between affective symptoms and BMI may differ according to gender and the onset of affective symptoms ${ }^{12}$. We also showed the association between SNP rs9939609 and BMI strengthened during childhood up to the age of 20 and then weakened again during adulthood ${ }^{19}$. The present study aimed first to investigate the possible bi-directional relationship between BMI and affective symptoms through the life course from adolescence to early old age. We hypothesized that, consistent with previous studies, any positive bi-directional relationship between increase of $\mathrm{BMI}$ and higher affective symptoms would be seen especially in women. We then investigated whether the FTO variant rs9939609 modifies the relationship between BMI and affective symptoms. We hypothesized that the rs9939609 obesity-related A allele would strengthen any positive relationships in an additive model.

\section{Methods \\ Participants}

The NSHD is a birth cohort study, initially consisting of a social class-weighted sample of 5362 children drawn from all single births within marriage during 1 week in March 1946 in England, Scotland, and Wales ${ }^{30,31}$. Blood samples for DNA extraction were collected from 2756 members at age 53 years and genotyping were conducted for 2498 participants.

Of 5362 cohort members, 4556 participants had at least one measure of BMI between ages 11 and 53 years (median number of measurements per individual $=5$, range $=1-7$ ), and of affective symptoms at ages 13 and $15,36,43,53$, and $60-64$ years (median $=3$, range $=1-5$ ); and are included in the initial analyses investigating the relationship between BMI and affective symptoms. Of these, 2469 participants also had the required information 
on BMI and affective symptoms and were included in the analysis assessing modification by the FTO gene. The participants included in the gene analysis were more likely to be female $(p<.001)$, had higher birth weight $(p=.012)$, higher overall cognitive score at age 8 years $(p<.001)$, and a lower adolescent emotional problem score $(p<.001)$ than those excluded.

The study was approved by Multi-Centre Research Ethics Committee for the survey at age 53 and by the Greater Manchester Local Research Ethics Committee and the Scotland Research Ethics Committee for the survey at age 60-64, and written informed consent was given by cohort participants.

\section{Measures}

\section{Body mass index}

Heights and weights were measured by school doctors at ages 11 and 15 years and by trained nurses using standardized protocols at ages 36,43 , and 53 years, and by self-reports at ages 20 and 26 years. BMI was calculated at each age using the standard weight $(\mathrm{kg}) /$ height $(\mathrm{m})^{2}$ formula.

\section{Affective symptoms}

Adolescent behavioural problems were rated at age 13 and 15 years by teachers using forerunners of the Rutter A scale $^{32,33}$. These ratings have been classified into three behavioural dimensions reflecting emotional (internalizing) problems (e.g., extremely fearful); conduct (externalizing) problems (e.g., a quarrelsome and aggressive child); and lack of self-control (e.g., a poor worker or lazy) ${ }^{34}$. An emotional problem score was calculated from the standardized sum of the relevant factor scores at both ages. Affective symptoms at age 36 years were assessed using a short version of the Present State Examination (PSE), a clinically validated interview administrated by trained nurses $^{35}$. We used the index of definition scale score (range $1-7)^{35}$. At age 43 years, the Psychiatric Symptom Frequency (PSF) scale was obtained with an 18-item self-reported questionnaire (range $0-90)^{36}$. At ages 53 and 60-64 years, the 28-item version of the General Health Questionnaire (GHQ-28) was adopted for self-reported depression and anxiety ${ }^{37}$. Each item was scored using a 4level Likert scale, and was recoded into 0-0-1-1. Therefore, the score ranged from 0 to 28 . Given the variation in assessment of affective symptoms, we used the continuous variables in analyses.

\section{Genotyping}

DNA was extracted and purified from whole blood using the Puregene DNA Isolation Kit (Flowgen, Leicestershire, UK), according to the manufacturer's protocol. The rs9939609 and rs1421085 SNPs were typed by Source Bioscience PLC using the Applied Biosystems (Foster
City, CA, USA) SNPlex technology which is based on an Oligonucleotide Ligation Assay combined with multiplex PCR amplification and capillary electrophoresis. Genotyping was performed using an ABI 3730xl DNA Analyser and ABI GeneMapper v4.0 software. The integrity of the genotyping was checked by genotypeing frequency, concordance between duplicates and Hardy-Weinberg equilibrium (HWE). The call rate was $99.5 \%$ with $>95 \%$ concordance between duplicate samples and there was no evidence of deviation from HWE $(p>.05)$. Since the SNP rs1421085 and rs9939609 were in the strong linkage disequilibrium $\left(r^{2}=0.89\right)$, our primary analysis focused on the rs9939609 polymorphism.

\section{Statistical analysis}

Our previous studies have indicated sex differences in the trajectory of both $\mathrm{BMI}^{19,38}$ and affective symptoms ${ }^{39}$, and the relationship between them ${ }^{12,40}$. Therefore, the analysis was stratified by sex if a following structural equation modelling (SEM) showed significant sex differences in BMI and affective symptoms, and interactions in the correlations between BMI and affective symptoms. First, a linear growth model was fitted to the BMI trajectory using four latent variables (intercept at age 11 years and changes from age 11-20 (adolescent), 20-36 (early adulthood), and 36-53 (midlife) years) within a SEM framework. Then we added the adolescent emotional problem score, allowing it to be correlated with the intercept of the BMI trajectory and with all latent variables representing BMI change; and the PSE score at 36 and PSF score at 43, allowing each to be correlated with BMI change from 36-53 (Fig. 1a). Each BMI variable was also related to all subsequent affective symptoms. Relationships between affective symptoms at all ages were assumed. Therefore, each relationship between BMI and affective symptom was tested considering previous measurement results of BMI and affective symptoms. Although BMI at age 60-64 years were available, previous analyses showed a reduction in the rate of BMI increase between age 53 and 60-64 years compared to between age 36 and 53 years $^{38}$. Therefore, to maintain a good statistical fit in a linear growth model with four latent variables, BMI at age 60-64 years was not included in the model. The estimation of the model was conducted using robust maximum likelihood estimation, and missing values were handled using full information maximum likelihood (FIML).

To test genetic modification of the associations between BMI and affective symptoms, the same model was fitted to the subsample with available genetic data and the FTO genotype was related to each of the four latent variables for BMI and the five observed affective symptoms variables, and the 20 gene by phenotype interactions were added (Fig. 1b). The rs9939609 A allele was considered a 


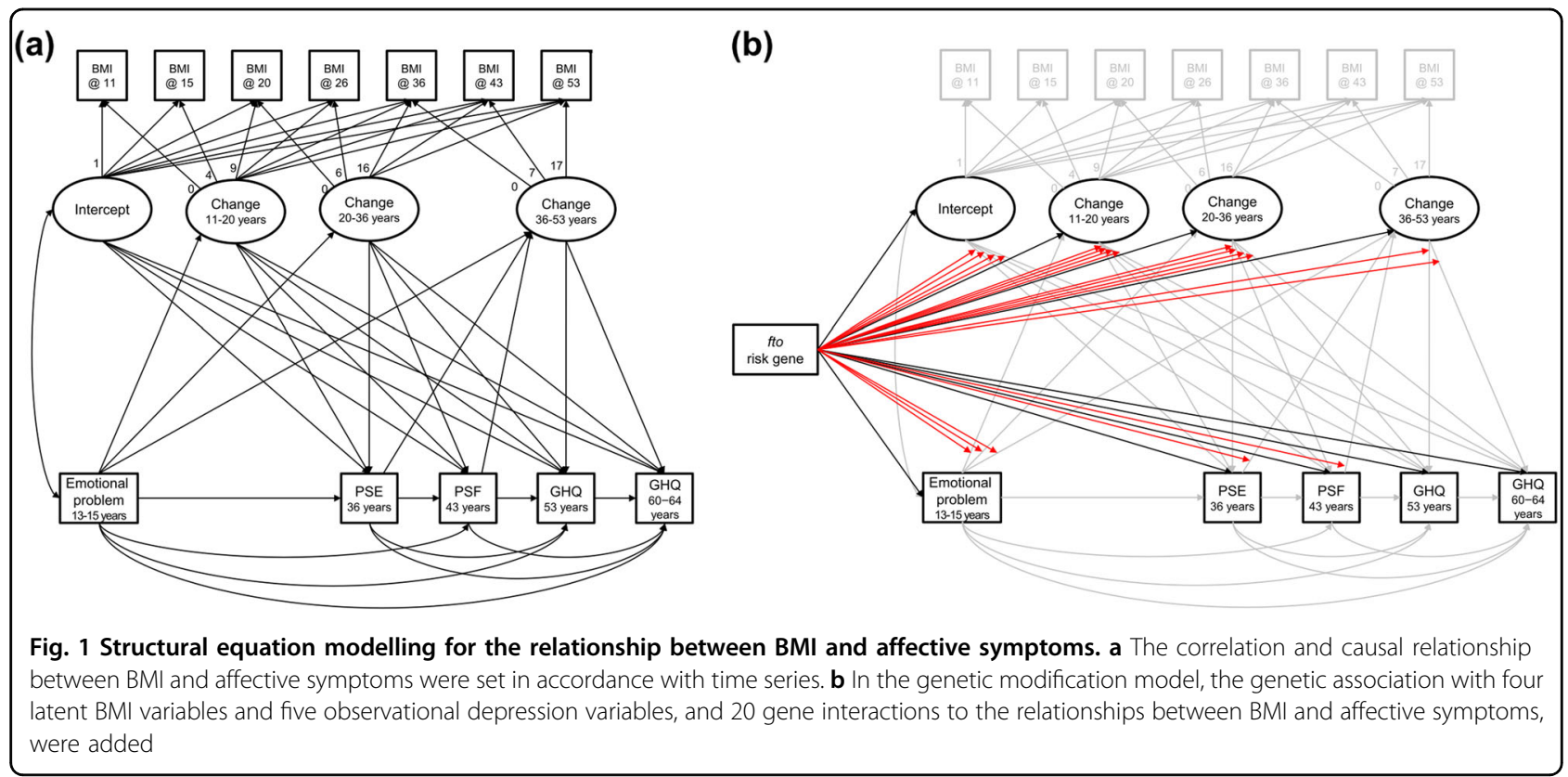

high risk for higher BMI and affective symptoms in an additive genetic model. Where there was evidence of effect modification, multiple-group analysis fitting the initial SEM model (Fig. 1a) was conducted to estimate the correlations between BMI and affective symptoms within each genotype group.

Statistical significance was set at $p<.05$. Good model fit was set at comparative fit index (CFI) $>.90$ and root mean square error of approximation (RMSEA) <.10. SEM was performed using the "lavaan" package version 0.5-16 in $\mathrm{R}$ version 3.1.1 $1^{41,42}$.

\section{Results}

Male participants had higher BMI compared to female at ages 11-43 years, and fewer affective symptoms at all ages (Table 1). Male participants were also more likely to have TA and less likely to have TT genotypes than female.

\section{Association between BMI and affective symptoms through the life course}

The initial SEM model for all participants had a good fit $(n=4556, \mathrm{CFI}=.919$, RMSEA $=.049)$. The model had the main effects of sex on BMI and affective symptoms, as well as sex interactions on the relationship between BMI and affective symptoms (Supplementary Fig. s1). Thus, further analyses were stratified by sex.

The SEM model specified in Fig. 1a had good fit in males $(n=2357$, CFI $=.969$, RMSEA $=.051)$ and females $(n=2199, \mathrm{CFI}=.920$, RMSEA $=.085)$. Figure 2 displays the significant correlations. In males, there was a negative correlation between BMI at age 11 years and adolescent emotional problems, but no other associations between
BMI and affective symptoms were significant at the $5 \%$ level (Fig. 2a). In females, adolescent emotional symptoms were positively associated with increases in BMI from ages 20 to 36 , and from ages 36 to 53 (Fig. 2b). In turn, increases in BMI from ages 20 to 36 were positively associated with GHQ depression and anxiety scores at age 53.

\section{Genetic modification of the relationship between BMI and affective symptoms}

The genetic modification model specified in Fig. $1 \mathrm{~b}$ had good fit in males $(n=1236, \mathrm{CFI}=.939, \mathrm{RMSEA}=.043)$ and females $(n=1233$, CFI $=.934$, RMSEA $=.045)$. Figure 3 displays the significant correlations. In both sexes, the rs9939609 A allele was associated with higher BMI at age 11 years, but not with subsequent rates of change (Fig. 3).

There were significant genetic interactions for both sexes; one in men and five in women (Fig. 3). In men, the negative correlation between greater BMI change between ages 20-36 and lower subsequent affective symptoms (at age 36) was stronger in the AA group than in the TA and TT groups (Fig. 4a). Among women, there was a positive relationship between BMI at 11 and GHQ score at 60-64 in the TT and TA groups but a negative association in the AA group (Fig. 4b). There was a strong negative association between BMI change between 20-36 and affective symptoms (at age 43) in the AA group but a positive association in the TT group (Fig. 4c). A similar positive association was observed between change in BMI over the same period and affective symptoms at 53 for the TT group, but there were null associations in 
Table 1 Characteristics of the analytic sample from the MRC NSHD

\begin{tabular}{|c|c|c|c|c|c|}
\hline & \multicolumn{2}{|l|}{ Male } & \multicolumn{2}{|c|}{ Female } & \multirow[t]{2}{*}{$p$-value ${ }^{b}$} \\
\hline & $n$ & Mean (SD) & $n$ & Mean (SD) & \\
\hline \multicolumn{6}{|l|}{ BMI $\left(\mathrm{kg} / \mathrm{m}^{2}\right)$} \\
\hline 11 years & 2050 & $17.3(2.1)$ & 1887 & $17.5(2.6)$ & 0.006 \\
\hline 15 years & 1881 & $19.6(2.4)$ & 1700 & $20.6(3.0)$ & $<.001$ \\
\hline 20 years & 1829 & $22.6(2.5)$ & 1735 & $21.8(2.9)$ & $<.001$ \\
\hline 26 years & 1822 & $23.4(2.8)$ & 1782 & $22.4(3.2)$ & $<.001$ \\
\hline 36 years & 1632 & $24.8(3.2)$ & 1648 & $23.6(4.1)$ & $<.001$ \\
\hline 43 years & 1617 & $25.7(3.5)$ & 1608 & $25.2(4.8)$ & $<.001$ \\
\hline 53 years & 1452 & $27.4(4.0)$ & 1496 & $27.4(5.5)$ & 0.81 \\
\hline \multicolumn{6}{|l|}{ Depressive symptoms } \\
\hline $\begin{array}{l}\text { Adolescent } \\
\text { emotional } \\
\text { problems }{ }^{\mathrm{a}}\end{array}$ & 2044 & $-0.09(0.96)$ & 1883 & $0.10(1.03)$ & $<.001$ \\
\hline $\begin{array}{l}\text { PSE score at age } \\
36 \text { years }\end{array}$ & 1640 & $1.7(1.1)$ & 1653 & $2.2(1.3)$ & $<.001$ \\
\hline $\begin{array}{l}\text { PSF score at age } \\
43 \text { years }\end{array}$ & 1600 & $9.6(10.0)$ & 1585 & $12.6(11.8)$ & $<.001$ \\
\hline $\begin{array}{l}\text { GHQ score at age } \\
53 \text { years }\end{array}$ & 1423 & $1.9(3.9)$ & 1479 & $3.2(4.9)$ & $<.001$ \\
\hline $\begin{array}{l}\text { GHQ score at age } \\
60-64 \text { years }\end{array}$ & 1048 & $1.7(3.1)$ & 1137 & $2.8(4.9)$ & $<.001$ \\
\hline $\begin{array}{l}\text { FTO rs9939609 } \\
\text { genotype, TT/TA/ } \\
\text { AA [\%] }\end{array}$ & 1238 & $\begin{array}{l}414 / 619 / \\
205[33.4 / \\
50.0 / 16.6]\end{array}$ & 1233 & $\begin{array}{l}460 / 534 / \\
239[37.3 / \\
43.3 / 19.4]\end{array}$ & $.004^{c}$ \\
\hline
\end{tabular}

$B M I$ body mass index, PSE a short version of the Present State Examination, PSF the Psychiatric Symptom Frequency scale, GHQ the 28-item version of the General Health Questionnaire, FTO fat mass and obesity-associated gene

a $Z$ scores from teacher-rated measures at age 13 and 15 years

${ }^{b}$ Gender differences were tested using $t$-tests, except for the genotype where chi-square tests were used

' Residual analysis showed that male participants were less likely to have $\Pi$ and more TA genotypes than female participants

the TA and AA groups (Fig. 4d). Similarly, greater changes in midlife BMI were associated with lower levels of symptoms at 53 in the TT group only (Fig. 4e). Finally, there was one suggestion of effect modification of an association from affective symptoms to BMI. The TT group showed a positive association between emotional problems in adolescence and greater change in BMI from 20-36 which was not observed in other genotype groups (Fig. 4f). The coefficients for the saturated models including all interactions with the FTO genotype are shown in Supplementary Tables S1-2.

The results for the SNP rs1421085 are provided in Supplementary Materials and Fig. S2. The results are largely similar except for the gene modification of the association between BMI change between 20-36 and affective symptoms at age 36 but not at age 43 in women.

\section{Discussion}

In a representative general population prospective cohort study we found that adolescent but not adult emotional problems were associated with BMI and that the relationships were different for men and women. A higher emotional problem score was associated with lower BMI at age 11 in men, while a higher emotional score was associated with greater midlife increases in BMI in women. In turn, greater midlife increases in BMI were associated with higher levels of affective symptoms at age 53 years in women. There was evidence that FTO rs9939609 modified these associations, particularly in women. In women greater increases in early adult BMI tended to be associated with lower levels of affective symptoms in the TT group (those with lower risk of overweight), whereas there were null or positive associations in the TA and AA groups, contrary to our hypothesis.

Strengths of this study include the use of a national population-based sample with repeated objective measurement of body height and weight, and well-validated assessment of affective symptoms through the life course. This means that we were able to assess the bi-directional relationship between BMI and affective symptoms across a 50-year period. To our knowledge, this is also the first study to report evidence of an effect modification of these associations by the FTO risk gene. The use of SEM and FIML meant that we were able to include a large sample size in the initial analysis investigating relationships between BMI and affective symptoms.

Several limitations should be considered. First, although the analytic approach used maximized the sample size, the disproportionate loss to follow-up of those of lower birthweight and from a less socially advantaged background may have affected the findings. However, in this cohort, higher birthweight is only weakly correlated with higher BMI in adulthood ${ }^{43}$ and lower birth weight is associated with adolescence but not adulthood affective symptoms ${ }^{44}$ and is therefore unlikely to have influences the findings to a great extent ${ }^{45}$. BMI is socially patterned according to both childhood and adult social class. Further, socioeconomic position and socially patterned health behaviours such as smoking and physical activity have been found to modify the association of genes with BMI, with the influence of genes being stronger in those from less advantaged groups and those with unhealthy behaviours $^{46,47}$. Hence, the differential loss of those from a more disadvantaged background who are likely to have higher BMI and more unhealthy behaviours may have led to an underestimate of associations in our study. More 

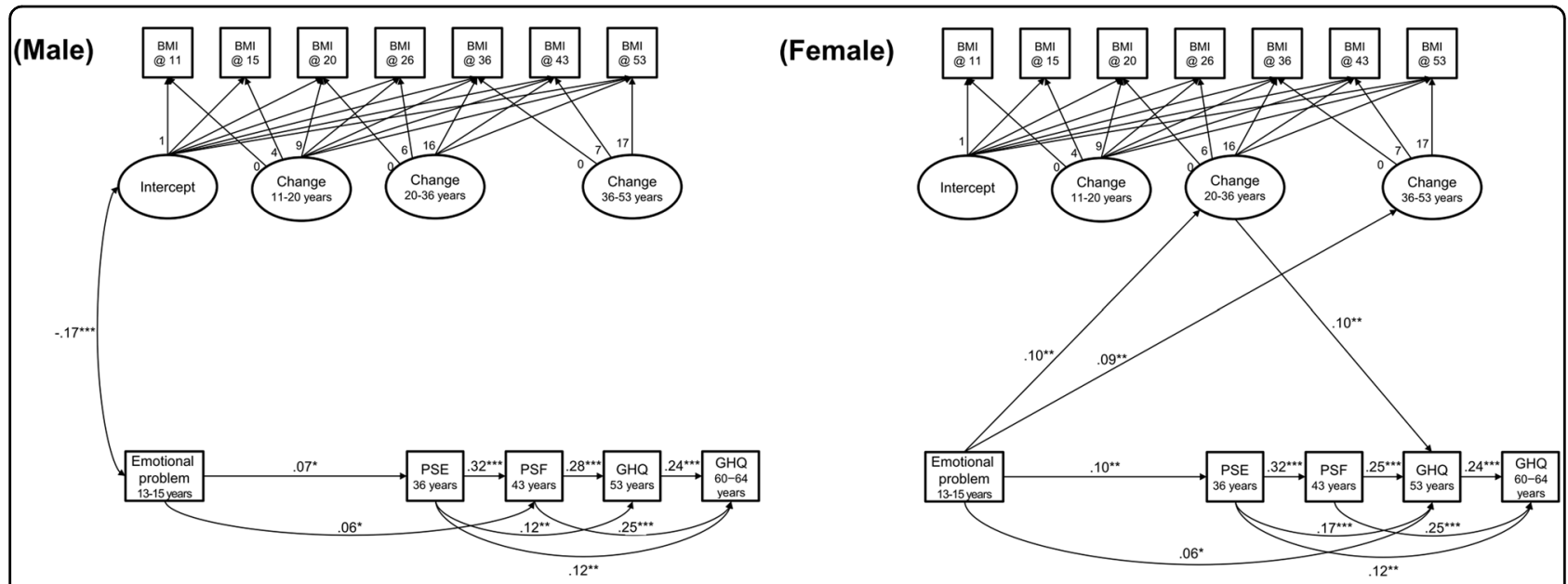

Fig. 2 The relationship between BMI and affective symptoms through the life course. Analysis was stratified by sex. Only significant relationships are displayed $\left({ }^{*} p<.05,{ }^{* *} p<.01,{ }^{* * *} p<.001\right)$

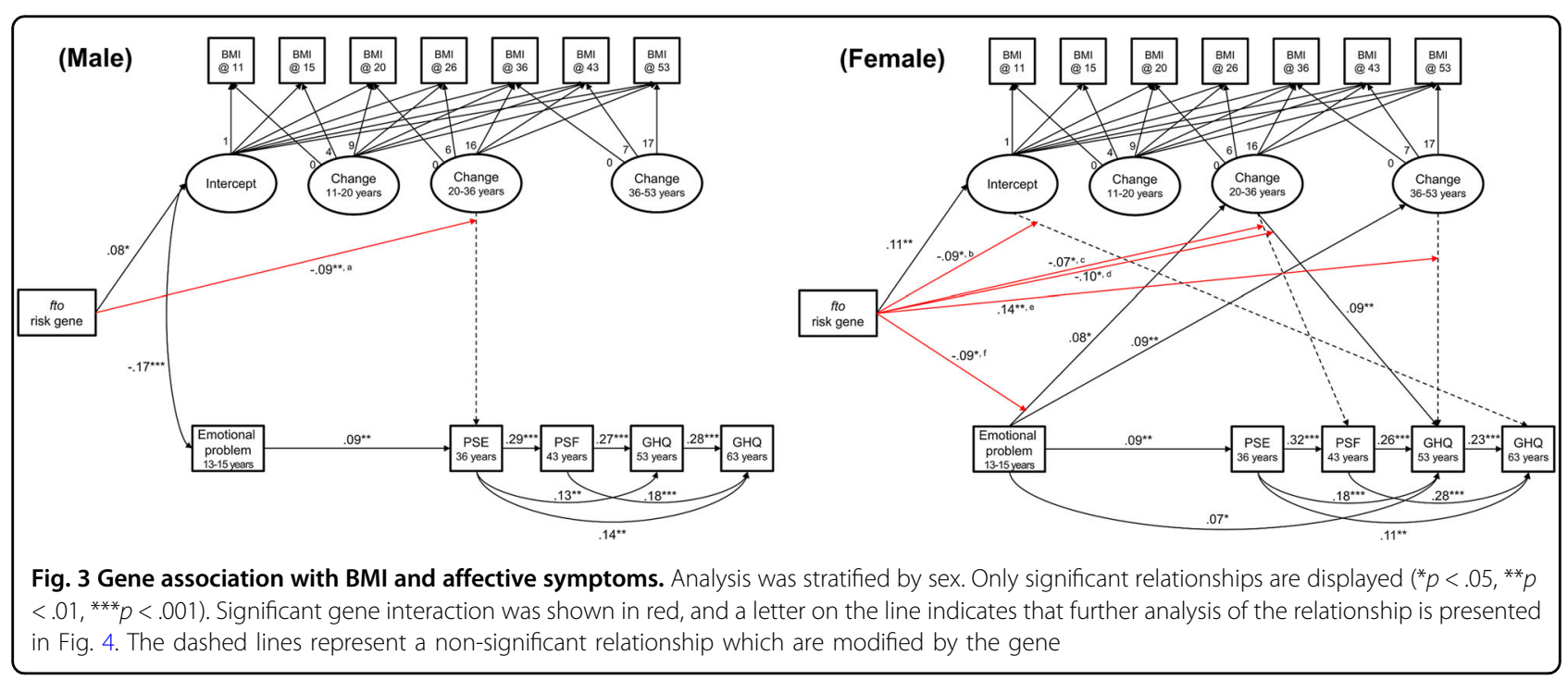

importantly, DNA was collected for the first time at age 53 years when 2989 participants were interviewed and hence the sample for the analyses including the FTO gene was reduced. In particular, the participants included in the genotype analysis had less adolescent emotional problems than those excluded, which may have resulted in a loss of statistical power in analyses. The higher cognitive capacity in those maintained in the analytic sample may also decrease the power to detect the relationship of interest since low childhood cognition is a risk factor for affective symptoms as well as increased body weight. In addition, some of those excluded have died from obesity-related diseases such as coronary heart disease. Therefore, we cannot rule out the possibility that the estimated relationships may be biased, but the missing data may mean that associations are underestimated. However, at age 53, the sample remained broadly representative of the general source population in Britain ${ }^{48}$ and there was little difference between the estimated coefficients from the initial SEM model on the maximum sample and the model on the sample with genotype information. Second, since the effect of the rs9939609 polymorphism on major depressive disorder has been found to vary by ethnicity ${ }^{28,29}$, the results may be different in samples containing other ethnic groups. However, the results from a large sample study including five different ethnicities were similar to our findings ${ }^{28,29}$. Third, although the scales used to measure affective symptoms at each age were well validated, the difference in scales used could influence the relative strengths of the relationships observed. Finally, we acknowledge that there may be alternative models to the theoretical model that we tested. For example, it may be 

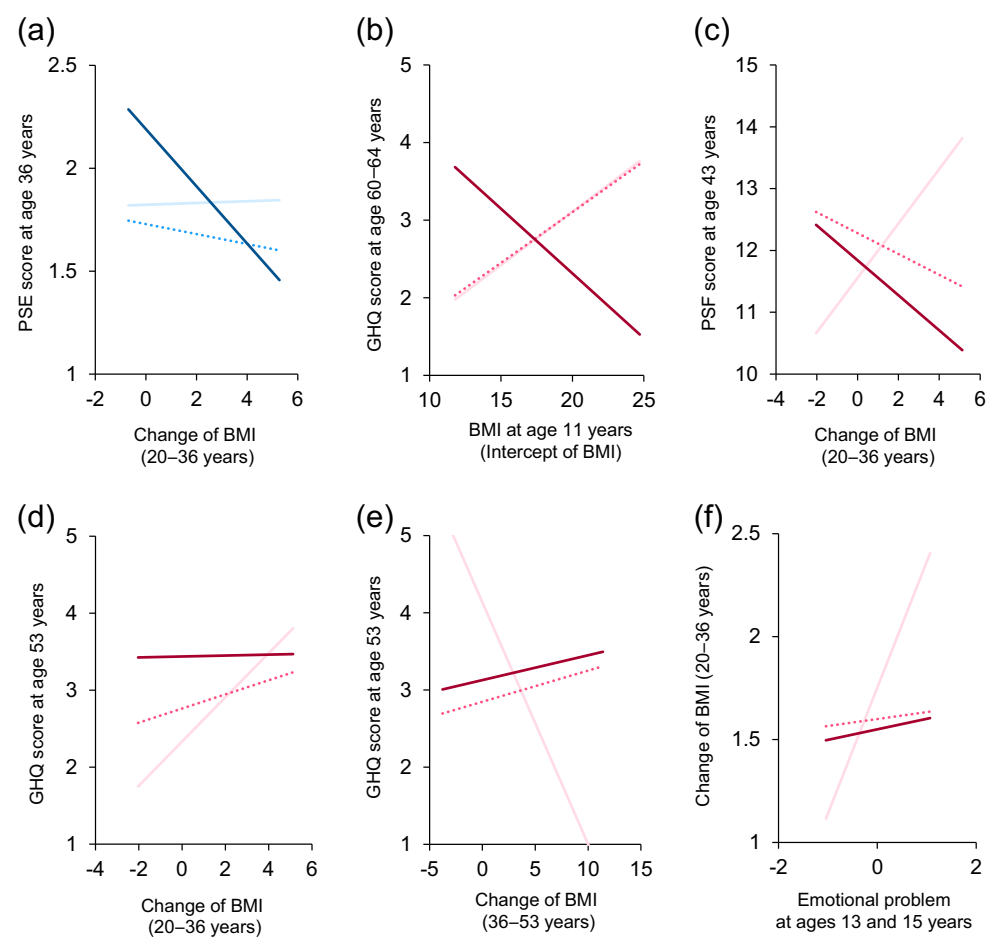

Fig. 4 Association between BMI and affective symptoms by FTO rs9939609 genotype. The results of multiple-group analysis for the initial SEM model (Fig. 1a) by FTO rs9939609 genotype are illustrated using equal loadings, means, residuals, and intercepts for the non-significant effect of genetic modification. In Fig. 4b, the line of $\Pi \mathrm{T}$ had an almost overlap with that of TA

that concurrent changes in symptoms, rather than prior symptoms, are associated with changes in BMI.

The present study found bi-directional associations between BMI and affective symptoms in females but not males. This reinforced the findings of large cross-sectional studies showing the relationship to be stronger in women than in men ${ }^{49-51}$. Previous longitudinal studies have shown that depressive episodes in adolescent girls were associated with obesity in adulthood ${ }^{10,52}$. One reason for these relationships being observed only in women may be because depressive symptoms are more associated with decreased physical activity and eating style disturbance in women than in men $^{52}$, which are also risk factors for obesity. Second, body image dissatisfaction, which is associated with affective symptoms, is more common in women than $\operatorname{men}^{53-55}$. A longitudinal study of the effect of weight-loss surgery showed that weight loss improved affective symptoms, in young women particularly ${ }^{56}$. Third, the experience of pregnancy and childbirth may influence this association. Adolescent depressive symptoms raise the risk for postpartum depression ${ }^{57}$, and are associated with weight retention after childbirth; ${ }^{58,59}$ and higher parity was associated with higher BMI at age 53 years in $\mathrm{NSHD}^{60}$. Finally, emotional stress responses and related hormone secretion in the HPA axis are greater in females ${ }^{7,53,61}$, which can influence weight increase. In addition, gonadal hormones influence both mood and body weight ${ }^{7,53}$.

The present study showed that the FTO rs9939609 risk variant exerted mostly protective effects in relation to affective symptoms for those with greater increases in BMI. Although the mechanism underlying these findings remains unknown, and these findings need to be replicated in view of the multiple interactions tested, one possible explanation may be that adult BMI is partially determined by differences in physical development in adolescence and young adulthood leading to differences in body composition. The FTO gene is associated with skeletal muscle as well as subcutaneous adipose tissue ${ }^{62}$, and $F T O$ deletion leads to a severe loss of muscle mass in humans and mice ${ }^{18}$. FTO mRNA expression is abundant in skeletal muscle in young adults compared to older people $^{62}$. In addition, FTO polymorphisms can modify the secretion of growth hormone ${ }^{63}$ and testosterone $e^{64}$, which are essential hormones for physical development and muscle growth. Thus, the FTO genotype-related increase in BMI in adolescence and young adulthood would be essential for physical development, which could lead to or result from better psychological health. Second, the rs9939609 A allele is protective of child emotional problems and attention deficits and hyperactivity disorder symptoms ${ }^{65}$, which is thought to be due to an altered 
reward response in dopaminergic neuron in the prefrontal cortex $^{66}$. Conversely, the mouse knock-out FTO gene within the midbrain dopaminergic system showed hyperactivity and aberrant sensitivity to dopamine stimulants ${ }^{23}$. Modification of the dopaminergic response in the prefrontal cortex by rs9939609 A carriers may be protective against the detrimental effect of increases in BMI on emergence of affective symptoms. Third, changes in BMI and affective symptoms for individuals with the protective allele could be more influenced by environmental factors such as health behaviours, while changes in those with the risk allele may be more influenced by genetic effect on their BMI. Thus unhealthy behaviours related to affective symptoms such as emotional eating and physical inactivity may be more influential in determining BMI among those with the protective allele. Previous studies have demonstrated modification of the association between genotype and BMI by health-related behaviours such as diet and physical activity ${ }^{20}$. There may be therefore more complex interactions between BMI change and health-related behaviours related to the risk gene and affective symptoms.

Our results showed that BMI and affective symptoms exhibit bi-directional positive relationships across the life course in women only. The FTO rs9939609 risk variant may be protective against affective symptoms in men, and particularly in women, who experience high increases BMI in adolescent and early adulthood. We have carried out multiple tests for effect modification of associations between BMI and affective symptoms by the FTO gene with inconsistent findings between men and women and between ages. The results should therefore be interpreted with some caution as they could be chance findings and require replication in other cohorts. The long-term effect might be caused by physical and psychological growth, along with the potential biological backgrounds such as hormonal and dopaminergic neuron system, rapidly occur during adolescence. Studies combining epidemiological investigation with biological measurement including gene polymorphism, muscle composition, and hormone concentration may be able to disentangle the complex relationship between BMI and affective symptoms and the FTO genotype according to age and gender.

\footnotetext{
Acknowledgements

S.K. was supported by the grants from MEXT KAKENHI Grant Number 17 H05921 and JSPS Strategic Young Researcher Overseas Visits Program for Accelerating Brain Circulation. A.W., R.H., and M.R. are funded by the UK Medical Research Council (Unit Programme numbers MC UU 12019/1; MC UU 12019/2; and MC UU 12019/4, respectively). The authors are grateful to NSHD study members for their continuing support. Data used in this publication are available upon request to the MRC National Survey of Health and Development Data Sharing Committee. Further details can be found at http:// www.nshd.mrc.ac.uk/data. https://doi.org/10.5522/NSHD/Q101; https://doi. org/10.5522/NSHD/Q102.
}

\section{Author details}

${ }^{1}$ MRC Unit for Lifelong Health and Ageing at University College London, 33 Bedford Place, London WC1B 5JU, UK. University of Tokyo Institute for Diversity \& Adaptation of Human Mind (UTIDAHM), 3-8-1 Komaba, Meguro-ku, Tokyo 153-8902, Japan. ${ }^{3}$ Center for Evolutionary Cognitive Sciences, Graduate School of Arts and Sciences, The University of Tokyo, 3-8-1 Komaba, Meguroku, Tokyo 153-8902, Japan

\section{Conflict of interest}

The authors declare that they have no conflict of interest.

\section{Publisher's note}

Springer Nature remains neutral with regard to jurisdictional claims in published maps and institutional affiliations.

Supplementary Information accompanies this paper at (https://doi.org/ 10.1038/s41398-018-0110-1).

Received: 19 April 2017 Revised: 9 November 2017 Accepted: 30 December 2017

Published online: 13 March 2018

\section{References}

1. Thapar, A., Collishaw, S., Pine, D. S. \& Thapar, A. K. Depression in adolescence. Lancet 379, 1056-1067 (2012).

2. Wang, Y. C., McPherson, K., Marsh, T., Gortmaker, S. L. \& Brown, M. Health and economic burden of the projected obesity trends in the USA and the UK. Lancet 378, 815-825 (2011).

3. Flegal, K. M., Kit, B. K., Orpana, H. \& Graubard, B. I. Association of all-cause mortality with overweight and obesity using standard body mass index categories: a systematic review and meta-analysis. J. Am. Med. Assoc. 309, 71-82 (2013).

4. Kupfer, D. J., Frank, E. \& Phillips, M. L. Major depressive disorder: new clinical, neurobiological, and treatment perspectives. Lancet 379, 1045-1055 (2012).

5. Flegal, K. M., Carroll, M. D., Kuczmarski, R. J. \& Johnson, C. L. Overweight and obesity in the United States: prevalence and trends, 1960-1994. Int. J. Obes. Relat. Metab. Disord. 22, 39-47 (1998).

6. Patton, G. C. et al. The prognosis of common mental disorders in adolescents: a 14-year prospective cohort study. Lancet 383, 1404-1411 (2014).

7. Bale, T. L. \& Epperson, C. N. Sex differences and stress across the lifespan. Nat. Neurosci. 18, 1413-1420 (2015).

8. Luppino, F. S. et al. Overweight, obesity, and depression: a systematic review and meta-analysis of longitudinal studies. Arch. Gen. Psychiatry 67, 220-229 (2010).

9. Puder, J. J. \& Munsch, S. Psychological correlates of childhood obesity. Int. J. Obes. 34, S37-S43 (2010).

10. Hasler, G. et al. Depressive symptoms during childhood and adult obesity: the Zurich Cohort study. Mol. Psychiatry 10, 842-850 (2005).

11. Pine, D. S., Goldstein, R. B., Wolk, S. \& Weissman, M. M. The association between childhood depression and adulthood body mass index. Pediatrics 107, 1049-1056 (2001).

12. Gaysina, D. et al. Symptoms of depression and anxiety, and change in body mass index from adolescence to adulthood: results from a British birth cohort. Psychol. Med. 41, 175-184 (2011).

13. Mansur, R. B., Brietzke, E. \& Mclntyre, R. S. Is there a "metabolic-mood syndrome"? A review of the relationship between obesity and mood disorders. Neurosci. Biobehav. Rev. 52, 89-104 (2015).

14. Preiss, K., Brennan, L. \& Clarke, D. A systematic review of variables associated with the relationship between obesity and depression. Obes. Rev. 14, 906-918 (2013).

15. Lopresti, A. L. \& Drummond, P. D. Obesity and psychiatric disorders: commonalities in dysregulated biological pathways and their implications for treatment. Prog. Neuropsychopharmacol. Biol. Psychiatry 45, 92-99 (2013).

16. Kuh, D., Cooper, R., Hardy, R., Richards, M. \& Ben-Shlomo, Y. A Life Course Approach to Healthy Ageing. (Oxford University Press, Oxford, 2014). 
17. Mezick, E. J., Hall, M. \& Matthews, K. A. Are sleep and depression independent or overlapping risk factors for cardiometabolic disease? Sleep Med. Rev. 15, 51-63 (2011).

18. Loos, R. J. \& Yeo, G. S. The bigger picture of FTO: the first GWAS-identified obesity gene. Nat. Rev. Endocrinol. 10, 51-61 (2014).

19. Hardy, R. et al. Life course variations in the associations between FTO and MC4R gene variants and body size. Hum. Mol. Genet. 19, 545-552 (2010)

20. Qi, Q. et al. FTO genetic variants, dietary intake and body mass index: insights from 177,330 individuals. Hum. Mol. Genet. 23, 6961-6972 (2014).

21. Livingstone, K. M. et al. FTO genotype and weight loss: systematic review and meta-analysis of 9563 individual participant data from eight randomised controlled trials. Br. Med. J. 354, i4707 (2016).

22. Zhao, X., Yang, Y., Sun, B. F., Zhao, Y. L. \& Yang, Y. G. FTO and obesity: mechanisms of association. Curr. Diab. Rep. 14, 486 (2014)

23. Hess, M. E. et al. The fat mass and obesity associated gene (Fto) regulates activity of the dopaminergic midbrain circuitry. Nat. Neurosci. 16, 1042-1048 (2013).

24. Fischer, J. et al. Inactivation of the Fto gene protects from obesity. Nature $\mathbf{4 5 8}$ 894-898 (2009).

25. Kivimaki, M. et al. Examining overweight and obesity as risk factors for common mental disorders using fat mass and obesity-associated (FTO) genotypeinstrumented analysis: the Whitehall II study, 1985-2004. Am. J. Epidemiol. 173 421-429 (2011)

26. Rivera, M. et al. Depressive disorder moderates the effect of the FTO gene on body mass index. Mol. Psychiatry 17, 604-611 (2012).

27. Rivera, M. et al. Interaction between the FTO gene, body mass index and depression: meta-analysis of 13701 individuals. Br. J. Psychiatry 211, 70-76 (2017).

28. Yao, Y. et al. Meta-analysis indicates that SNP rs9939609 within FTO is not associated with major depressive disorder (MDD) in Asian population. J. Affect. Disord. 193, 27-30 (2016).

29. Samaan, Z. et al. The protective effect of the obesity-associated rs9939609 A variant in fat mass- and obesity-associated gene on depression. Mol. Psychiatry 18, 1281-1286 (2013).

30. Kuh, D. et al. Cohort profile: updating the cohort profile for the MRC National Survey of Health and Development: a new clinic-based data collection for ageing research. Int. J. Epidemiol. 40, e1-e9 (2011).

31. Wadsworth, M., Kuh, D., Richards, M. \& Hardy, R. Cohort profile: the 1946 National Birth Cohort (MRC National Survey of Health and Development). Int. J. Epidemiol. 35, 49-54 (2006).

32. Elander, J. \& Rutter, M. Use and development of the Rutter parents' and teachers' scale. Int. J. Methods Psychiatr. Res. 6, 63-78 (1996)

33. Rutter, M., Tizard, J. \& Whitmore, K. Education, Health and Behaviour. (Longman Publishing Group, London, 1970).

34. $\mathrm{Xu}, \mathrm{M}$. K. et al. Adolescent self-organization predicts midlife memory in a prospective birth cohort study. Psychol. Aging 28, 958-968 (2013).

35. Wing, J. K., Cooper, J. E. \& Sartorius, N. The Measurement and Classification of Psychiatric Symptoms. (Cambridge University Press, Cambridge, 1974).

36. Lindelow, M., Hardy, R. \& Rodgers, B. Development of a scale to measure symptoms of anxiety and depression in the general UK population: the psychiatric symptom frequency scale. J. Epidemiol. Community Health 51, 549-557 (1997).

37. Goldberg, D. P. \& Hillier, V. F. A scaled version of the general health questionnaire. Psychol. Med. 9, 139-145 (1979).

38. Koike, S., Hardy, R. \& Richards, M. Adolescent self control behavior predict body weight through the life course: a prospective birth cohort study. Int. J. Obes. 40, 71-76 (2016).

39. Hatch, S. L. et al. Childhood cognitive ability and adult mental health in the British 1946 birth cohort. Soc. Sci. Med. 64, 2285-2296 (2007).

40. Gaysina, D. et al. Association between adolescent emotional problems and metabolic syndrome: the modifying effect of C-reactive protein gene (CRP) polymorphisms. Brain Behav. Immun. 25, 750-758 (2011).

41. R Foundation for Statistical Computing. R: a language and environment for statistical computing. http://www.R-project.org/ (2014).

42. Rosseel, Y. lavaan: an R package for structural equation modeling. J. Stat. Soft 48, 1-36 (2012).
43. Hardy, R. et al. Birthweight and blood pressure in five European birth cohort studies: an investigation of confounding factors. Eur. J. Public Health 16, 21-30 (2006).

44. Colman, I. et al. Early development, stress and depression across the life course: pathways to depression in a national British birth cohort. Psychol. Med. 44, 2845-2854 (2014).

45. Wojcik, W., Lee, W., Colman, I., Hardy, R. \& Hotopf, M. Foetal origins of depression? A systematic review and meta-analysis of low birth weight and later depression. Psychol. Med. 43, 1-12 (2013).

46. Dinescu, D., Horn, E. E., Duncan, G. \& Turkheimer, E. Socioeconomic modifiers of genetic and environmental influences on body mass index in adult twins. Health Psychol. 35, 157-166 (2016).

47. Johnson, W. et al. Modification of genetic influences on adiposity between 36 and 63 years of age by physical activity and smoking in the 1946 British Birth Cohort study. Nutr. Diabetes 4, e136 (2014).

48. Wadsworth, M. E. et al. The life course prospective design: an example of benefits and problems associated with study longevity. Soc. Sci. Med. 57 2193-2205 (2003)

49. UI-Haq, Z. et al. Gender differences in the association between adiposity and probable major depression: a cross-sectional study of 140,564 UK Biobank participants. BMC Psychiatry 14, 153 (2014).

50. Atlantis, E. \& Baker, M. Obesity effects on depression: systematic review of epidemiological studies. Int. J. Obes. 32, 881-891 (2008)

51. Heo, M., Pietrobelli, A., Fontaine, K. R., Sirey, J. A. \& Faith, M. S. Depressive mood and obesity in US adults: comparison and moderation by sex, age, and race. Int. J. Obes. 30, 513-519 (2006)

52. Richardson, L. P. et al. A longitudinal evaluation of adolescent depression and adult obesity. Arch. Pediatr. Adolesc. Med. 157, 739-745 (2003)

53. Hyde, J. S., Mezulis, A. H. \& Abramson, L. Y. The ABCs of depression: integrating affective, biological, and cognitive models to explain the emergence of the gender difference in depression. Psychol. Rev. 115, 291-313 (2008).

54. Stice, E., Hayward, C., Cameron, R. P., Killen, J. D. \& Taylor, C. B. Body-image and eating disturbances predict onset of depression among female adolescents: a longitudinal study. J. Abnorm. Psychol. 109, 438-444 (2000).

55. Kostanski, M. \& Gullone, E. Adolescent body image dissatisfaction: relationships with self-esteem, anxiety, and depression controlling for body mass. J. Child Psychol. Psychiatry 39, 255-262 (1998).

56. Dixon, J. B., Dixon, M. E. \& O'Brien, P. E. Depression in association with severe obesity: changes with weight loss. Arch. Intern. Med. 163, 2058-2065 (2003).

57. Norhayati, M. N., Nik Hazlina, N. H., Asrenee, A. R. \& Wan Emilin, W. M. Magnitude and risk factors for postpartum symptoms: a literature review. J. Affect. Disord. 175C, 34-52 (2014)

58. Xiao, R. S. et al. The impact of sleep, stress, and depression on postpartum weight retention: a systematic review. J. Psychosom. Res. 77, 351-358 (2014).

59. Herring, S. J. et al. Association of postpartum depression with weight retention 1 year after childbirth. Obesity 16, 1296-1301 (2008).

60. Hardy, R., Lawlor, D. A., Black, S., Wadsworth, M. E. \& Kuh, D. Number of children and coronary heart disease risk factors in men and women from a British birth cohort. BJOG 114, 721-730 (2007).

61. Piccinelli, M. \& Wilkinson, G. Gender differences in depression: critical review. Br. J. Psychiatry 177, 486-492 (2000)

62. Grunnet, L. G. et al. Regulation and function of FTO mRNA expression in human skeletal muscle and subcutaneous adipose tissue. Diabetes $\mathbf{5 8}$ 2402-2408 (2009).

63. Rosskopf, D. et al. The growth hormone--|GF-I axis as a mediator for the association between FTO variants and body mass index: results of the Study of Health in Pomerania. Int. J. Obes. 35, 364-372 (2011).

64. Wehr, E. et al. Association of FTO gene with hyperandrogenemia and metabolic parameters in women with polycystic ovary syndrome. Metabolism 59 575-580 (2010).

65. Velders, F. P. et al. FTO atrs9939609, food responsiveness, emotional contro and symptoms of ADHD in preschool children. PLOS ONE 7, e49131 (2012).

66. Ishii-Takahashi, A. et al. Neuroimaging-aided prediction of the effect of methylphenidate in children with attention-deficit hyperactivity disorder: a randomized controlled trial. Neuropsychopharmacology 40, 2676-2685 (2015). 